\title{
Use of security patterns for development of secure healthcare information system
}

\begin{abstract}
Healthcare systems have recently received attention from industry related to the sensitive and confidential information stored in it. This information is shared among different physicians, surgeons and technicians in the distributed and heterogeneous environment. Bearing these security concerns in mind a model driven methodology of using security patterns for development of secure healthcare information systems is proposed. This methodology consists of an extension of Unified Modeling Language (UML) deployment diagram namely as Security Patterns Deployment Diagram (SPDD) and a tool that supports for extending the use of proposed diagram. The proposed method has been validated by performing a case study on Healthcare Information System.
\end{abstract}

Keyword: Healtcare system security; Secure software architecture; Security patterns application; UML 